\title{
Una nueva especie de Salvia (Lamiaceae) del Norte de Perú
}

\author{
A new species of Salvia (Lamiaceae) from Northern Peru
}

\begin{abstract}
1 Herbarium Truxillense (HUT), Universidad Nacional de Trujillo. Jr. San Martín 392. Trujillo, Perú. $\dagger$

2 Herbarium Truxillense (HUT), Universidad Nacional de Trujillo. Jr. San Martín 392. Trujillo, Perú. Email Eric F. Rodríguez Rodríguez: efrr@unitru.edu.pe
\end{abstract}

Presentado: $\quad$ 07/05/2012 Aceptado: 18/08/2012 Publicado online: 10/11/2012

\section{Abundio Sagástegui Alva ${ }^{1} \dagger$ y Eric F. Rodríguez Rodríguez ${ }^{2}$}

\section{Resumen}

Se describe e ilustra una nueva especie de Salvia L. (Lamiaceae), denominada Salvia vargas-llosae Sagást. \& E. Rodr. sp. nov. perteneciente a la sección Cylindriflorae (Epling) Epling, procedente de los andes del Norte del Perú (provincia Celendín, departamento Cajamarca), típica de la jalca Sendamal-Kumulca-Challuayaco sobre los $3000 \mathrm{~m}$ de altitud y aparentemente endémica a esta parte del país. Se discute con sus relacionadas y adicionalmente se presentan datos sobre su distribución geográfica, ecológica y estado de conservación.

Palabras clave: Salvia, Cylindriflorae, Lamiaceae, especie nueva, Norte del Perú.

Abstract

Salvia vargas-Ilosae Sagást. \& E. Rodr. sp. nov. is described and illustrated as a new species of Salvia L. (Lamiaceae) from the Andes of Northern Peru (province of Celendin, department of Cajamarca). This new species belongs to the section Cylindriflorae (Epling) Epling and typically restricted to the region jalca (SendamalKumulca-Challuayaco) above the $3000 \mathrm{~m}$ of altitude and apparently endemic to this part of the country. It is discussed with its closest relatives and additionally data on its geographical distribution, ecology and state of conservation are provided.
\end{abstract}

Key words: Salvia, Cylindriflorae, Lamiaceae, new species, Northern Peru.

\section{Introducción}

El género cosmopolita Salvia L., el más extenso de la familia Lamiaceae (=Labiatae), cuenta en la actualidad con unas 935 especies reconocidas (Fernández-Alonso 2008 a, b). Para la flora del Perú se han registrado 79 taxones entre especies y subespecies, siendo catalogado como el más diverso de la familia (Zarucchi 1993, Granda 2010). Actualmente, son consideradas a 32 especies endémicas, constituyéndose en el género más rico en endemismos de la familia (Rodríguez 2006, Granda 2010). Los últimos estudios que se han efectuado con material peruano son los realizados por Epling \& Játiva $(1963,1966,1968)$ y Granda (2010). La mayoría de especies presentes en el Perú se encuentran registradas para el norte. Especialmente los andes constituyen un importante hábitat. Revisando críticamente tanto el material de herbario como el de las últimas colecciones procedentes de los andes peruanos, en particular del Norte, se ha encontrado una nueva especie que denominamos Salvia vargas-llosae Sagást. \& E. Rodr. sp.nov. procedente de la cadena central de los andes del norte en la zona de Cajamarca, Prov. Celendín. Se debe enfatizar que de esta cadena central norteńa, perteneciente a la zona de Amotape-Huancabamba de elevada riqueza endémica (Weigend 2002, 2004), se han descrito nuevos taxones para la ciencia de material recolectado por personal del Herbarium Truxillense (HUT) de la Universidad Nacional de Trujillo (e.g.: Salvia lanicaulis Epling \& Játiva, S. medusa Epling \& Játiva, Salvia xanthophylla Epling \& Játiva) (Epling \& Játiva 1963). Consecuentemente, la descripción, ilustración y discusión de este nuevo taxón es el objetivo de este trabajo.

\section{Material y métodos}

El estudio está basado en la revisión de material de herbario (CPUN, HUT, F, MO) y observaciones directas de hábito y hábitat en el campo. Las colecciones se realizaron de acuerdo con la metodología y técnicas convencionales de herborización (Rodríguez \& Rojas 2002). Adicionalmente al trabajo de campo se fijó y conservó material en líquido (alcohol etílico al 70\% o AFA) para estudiar la estructura floral. El material botánico del tipo fue depositado en los siguientes herbarios: HUT, F, MO. Son presentadas, la descripción, discusión, delineación y mediciones de la especie (Fig. 1 A-H). Se adicionan datos sobre su distribución geográfica, ecología, fenología y estado de conservación según criterios de la Lista Roja UICN (UICN 2001). Los acrónimos de los herbarios son citados según Holmgren et al. (1990).

\section{Taxonomía}

Salvia vargas-Ilosae Sagást. \& E. Rodr. sp.nov.

(Fig. 1 A-H)

Tipo: PERÚ. Dpto. Cajamarca, Provincia Celendín, Challuayaco (La Encañada-Celendín), ladera rocosa, $3600 \mathrm{~m}, 18$ mayo 1976, A. Sagástegui A., J. Cabanillas S. \& O. Dios C. 8409 (Holótipo: HUT-13705; Isótipos: F, MO).

Herba perenne, ca. $50 \mathrm{~cm}$ alta, erecta vel ascendens, parce ramosa, rhizomatosa. Caulis tetragonis, 2-3 mm crassis, pilis septatis aglandularis, ca. $1 \mathrm{~mm}$ longis et pilis septatis glandularis, ca. 0,5 $\mathrm{mm}$ longis. Folia opposita, petiolata; petiolo acanalato; lamina ovata, basi cordata, apice obtusa, crenata, 16-22 × 14-20 mm. Inflorescentiae racemosae terminales, (5-) 7-15 (-17), verticillastrae 4-7(-8) florae. Bracteis late ovato-ellipticis, acuminatis, caducis, fuscescentibus, externe pilosis. Calyx bilabiatis, 10-13 × 5-6 mm; superne labium 4-6 $\times$ 3-3,5 mm, 6-nervatis, inferne labium 2-lobatum, lobulis breviter apiculatis, 4-5 × 2-3 mm, 3-nervatis. Corolla violacea, tubo 18-20 mm longo, superne labium 7-8 $\mathrm{mm}$ longum, inferne labium 12-15 mm longum, 3-lobulatum, latere lobulis ellipticis, 2-3 × $2 \mathrm{~mm}$, médium 2-lobulatis, 5-6 × 6-8 mm. Filamentum adnatum ca. $15 \mathrm{~mm}$ ad basim tubular; antherae basifijae. Ovarium ellipsoideum, $2 \times 1,2 \mathrm{~mm}$; stylis bifidus. Nucula ellipsoide, fuscescentibus, maculata, 2,5-3 × 1,5 mm. 



Figura 1. Salvia vargas-Ilosae Sagást. \& E. Rodr. A. Rama florífera; B. Hoja; C. Flor; D. Bráctea de la parte media de la inflorescencia; E. Estambres mostrando al filamento, conectivo y anteras; F. Ovario, estilo y estigmas; G. Disposición de las núculas; H. Núcula. Delineado por R. Aguirre T. del holótipo: A. Sagástegui A., J. Cabanillas S. \& O. Dios C. 8409 (HUT). 
Hierba perenne de hasta $50 \mathrm{~cm}$ de altura, erecta, decumbente o ascendente, estolonífera, poco ramificada, solamente en la parte inferior, ramitas hasta $7 \mathrm{~cm}$ de largo; indumento blanquecino piloso-glanduloso, con pelos simples septados aglandulares, 4-7 células, célula basal buliforme, de hasta $1 \mathrm{~mm}$ de largo y pelos simples septados con ápice glandular, 3-4 células, de hasta 0,5 $\mathrm{mm}$ de largo. Tallos de 2-3 mm de diámetro, tetrágonos, generalmente rojizos, con indumento concentrado en la zona media de las caras y escaso en las aristas, constituido mayormente por pelos glandulares; entrenudos 10-65 (-75) mm de longitud. Hojas opuestas, pecioladas (pecíolo canalado, 10-15 mm de longitud, pubescencia abundante constituida principalmente por pelos simples aglandulares), láminas ovadas, ápice obtuso, base cordada, crenadas (dientes subredondeados e irregulares, 10-12 a cada lado), con 3-4 nervios secundarios a cada lado del nervio medio, nervios de tercer y cuarto orden poligonal, nerviación poco resaltada en el envés; haz verde oscuro y envés verde claro, pubescentes en la haz, excepto en las nervaduras, indumento constituido de pelos simples septados aglandulares, igual indumento pero más corto y disperso en las nervaduras del envés; de $16-22 \times 14-20 \mathrm{~mm}$ en las mas grandes. Inflorescencia terminal, racemosa, interrumpida, $80-180 \mathrm{~mm}$ de largo, constituida por (5-) 7-15 (-17) verticilastros, 4-7(-8) flores (antesis asincrónica), separados 5-40 mm entre sí (menor separación hacia el ápice), parte basal del raquis sin flores de 45$110 \mathrm{~mm}$, densamente hírtulo, con indumento generalmente no glandular; brácteas ampliamente ovado-elípticas, acuminadas, caducas, marrones, exteriormente pubescentes, dominancia de pelos simples no glandulares sobre los glandulares, 8,5-9 × 5,5$6 \mathrm{~mm}$; pedicelos de 5-6 $\mathrm{mm}$ de longitud en la antesis y 8-11 $\mathrm{mm}$ en la fructificación, cortamente hírtulos, con indumento simple septado no glandular. Cáliz bilabiado, de $10-13 \mathrm{~mm}$ de longitud y 5-6 mm de ancho en la antesis, 14-18 $\times$ 6-7 mm en fructificación, tubo 7-10 $\mathrm{mm}$ de largo, externamente con indumento corto, hirto, dispuesto en los nervios, predominancia de pelos glandulares uniformemente distribuidos, internamente glabros; labios muy abiertos, desiguales, el superior de 4-6 mm de longitud y 3-3,5 mm de ancho, con 6 nervios conspicuos de color morado intenso, el inferior bidentado, dientes brevemente apiculados, de 4-5 × 2-3 mm, con 3 nervios conspicuos verdes cada uno. Corola violácea, indumento abundante formado por pelos simples no glandulares septados lanuginosos, violáceos, de hasta $0,5 \mathrm{~mm}$ de longitud; tubo de la corola de $18-20 \mathrm{~mm}$, recto y gradualmente ensanchado desde la base (3-3,5 mm de anchura) hasta el ápice (6 $\mathrm{mm}$ de anchura); labio superior de 7-8 mm de longitud, labio inferior amplio, patente, $12-15 \mathrm{~mm}$ de largo, 3-lobulado, lóbulos laterales elípticos, $2-3 \times 2 \mathrm{~mm}$, lóbulo medio más amplio, escotado, 2-lobulado, 5-6 × 6-8 mm, sobrepasando ca. 2-3 mm en longitud a los laterales. Estambres con filamentos adnatos a ca. $15 \mathrm{~mm}$ de la base del tubo, rojizos, glabros, de $4 \mathrm{~mm}$ de largo; conectivo levemente arqueado, rojizo, de $20 \mathrm{~mm}$ de longitud, sobrepasando 10-12 $\mathrm{mm}$ al labio superior; anteras estrechamente cilíndricas, basifijas, oscuras, polen amarillo, de $3 \mathrm{~mm}$. Ovario elipsoidal, amarillento, $2 \times$ 1,2 mm; estilo de 35-40 mm de longitud, rojizo, glabro, con ramas estigmáticas desiguales, la superior de $3 \mathrm{~mm}$ y la inferior de 1-1,5 mm, divergentes, claramente ensanchado-espatulada y obtusa en el extremo distal. Núculas elipsoidales, de color marrón claro con máculas marrón oscuro y contornos redondeados, de 2,5-3 × 1,5 mm.

\section{Material Adicional Examinado:}

PERU: Dpto. Cajamarca. Prov. Celendín: Chaluayaco, entre Kumulca y Celendín, 3350 m, 4 Mayo 1970, I. Sánchez V. \& W. Ruiz V. 469 (CPUN-3192). En el lugar llamado Challuayaco, sobre la carretera a Celendín, al este del paso de Cumullca, 3550 m, 12 Mayo 1984, I. Sánchez V. \&W. Ruiz V. 3474 (CPUN-3140). Distr. Encañada, Cumulca, 7 Abril 1966, I. Sánchez V. \& M. Alva Z.189 (CPUN-3166). Sendamal, entre la Encañada y Celendín, 3330 m, 3 Julio 1975, I. Sánchez V. \& W. Ruiz V. \& J. Sánchez V. 1691 (CPUN-1691). Challuayaco (Encañada-Celendín), 3350 m, 4 Mayo 1970, A. Sagástegui A. 7388 (HUT-7402). Jalca de Kumulca, 3500 m, 01 Julio 1978, A. López M. \& A. Aldave P. 8452 (HUT-15381).

\section{Discusión Taxonómica:}

Salvia vargas-llosae Sagást. \& E. Rodr. sp.nov. se encuentra circunscrita en la Sección Cylindriflorae (Epling) Epling principalmente porque es una hierba perenne típica de los Andes con 4-7(-8) flores en cada verticilastro, corola desde rojiza hasta violácea, parte interna del tubo de la corola sin indumento ni papilas, estambres bien exertos y adnatos sobre la mitad del tubo de la corola, así como por presentar estilos glabros. De todas las especies de la Sección con la que presenta mayor afinidad es con S. ayavacensis Kunth (= Salvia mucidistachys Epling) (ver Bonpland \& Humboldt 1817, Macbride 1960, Zarucchi 1993), por el tipo de indumento, forma y margen de las hojas, número de flores por verticilastro, color de flores, tamaño del filamento del estambre, entre otras características. Sin embargo, $S$. ayavacensis se diferencia claramente de la nueva especie porque es una planta robusta (mayor a $1 \mathrm{~m}$ versus hasta $50 \mathrm{~cm}$ de alto), hojas mas grandes y pecíolos mas largos, disposición de los verticilastros en la inflorescencia, flores más grandes (e.g.: cáliz de $17 \mathrm{~mm}$ versus $10-13 \mathrm{~mm}$ de largo en antesis, tubo de la corola ca. $3 \mathrm{~cm}$ versus $2 \mathrm{~cm}$ de largo, conectivo del estambre ca. $40 \mathrm{~mm}$ de largo versus $20 \mathrm{~mm}$ de largo en la especie nueva), y pedicelos florales en antesis son ca. 2 veces y media mas largos a los ocurridos en S. vargas-llosae (15-16 mm versus 5-6 mm). Así mismo, $S$. ayavacensis es una especie endémica al departamento de Piura (Rodríguez 2006) [cerca al río Cutaco, prov. Ayavacatipo, y Talaneo-Jalca Cixse, prov. Huancabamba, ver colección: A. Sagastegui A. \&J. Cabanillas S. 8634 (HUT-13961)] y que crece en los Andes de la vertiente occidental norteña a altitudes más bajas (bajo los 3,000 m de altitud)en relación con la especie nueva. En general $S$. vargas-llosae es la especie que presenta las flores más pequeñas de la Sección. Se debe precisar que con Salvia lanicaulis Epling \& Játiva [ver colecciones de jalca Kumulca: A. Sagástegui A., J. Mostacero L. \& S. Leiva G. 12039 (HUT19712), A. Sagástegui A., J. Cabanillas S. \& O. Dios C. 8113 (HUT-13210), otra especie de la misma Sección, comparten solamente la distribución geográfica y hábitat (Jalca) a mas de $3000 \mathrm{~m}$. Así mismo, otras especies norteñas pertenecientes a la misma Sección, como $S$. cylindriflora Epling y S. hirta Kunth de Piura, y $S$. heerii Regel de Cajamarca, crecen en diferentes hábitats y a altitudes mucho más bajas, pero mayormente carecen de afinidad con el nuevo taxón.

\section{Distribución, Ecología y Fenología:}

Salvia vargas-llosae es aparentemente endémica de la jalca de la Provincia Celendín, Cajamarca (localidades de Sendamal, Kumulca y Challuayaco), distribuyéndose arriba de los 3,000 
m de altitud. Es una de las pocas especies andinas de Salvia que crece en altitudes elevadas. En las localidades mencionadas es encontrada con relativa frecuencia formando poblaciones de pocos individuos. Habita laderas pedregosas expuestas, suelos negros de humificación variable, musgosos, así como en ambientes con humedad edáfica; asociada con hierbas, arbustos y vegetación propia del área que incluye a Salvia lanicaulis Epling \& Játiva. La nueva especie está restringida a la denominada zona de Amotape-Huancabamba de elevada riqueza endémica (Weigend 2002, 2004).

Florece y fructifica en mayo después de las lluvias. Es visitada por colibríes e insectos (planta melífera).

\section{Etimología:}

La nominación de este taxón nuevo para la ciencia la asociamos con el Premio Nobel de Literatura 2010 el peruano Dr. Mario Vargas Llosa para felicitarlo y adherirnos a su prestigio universal, a quien rogamos acepte esta forma de hacerlo en calidad de Académicos y Científicos de la Universidad Nacional de Trujillo, Perú, en donde por felicidad nos ocupa la Scientia Amabilis, es decir el estudio de las plantas que son los seres que determinan la vida en el mundo.

\section{Estado de Conservación:}

La nueva entidad hasta el momento es considerada endémica al norte del Perú y actualmente su distribución se encuentra restringida a la jalca de la provincia Celendín, Cajamarca. Según las colecciones efectuadas generalmente en el mismo lugar, el área de presencia y ocupación es relativamente pequeña, el número y tamaño de sus poblaciones son pequeñas y dispersas. Además, la preocupación esta focalizada en que esta especie vive en ecosistemas frágiles que no están protegidos por el Estado peruano y en donde es notoria la pérdida y alteración progresiva de hábitat (e.g.: quemas periódicas, pastoreo, cultivo de coníferas como Pinus radiata D. Don.). De acuerdo con esta información y según los criterios de la Lista Roja UICN (UICN, 2001), debería ser incluida en la categoría VU (Vulnerable).

\section{Agradecimientos}

Agradecemos a nuestro maestro Dr. Arnaldo López Miranda (HUT) por sus enseñanzas y dirigir los trabajos de campo en el Norte del Perú; a la Dra. Nancy Hensold (F) por advertir la presencia de esta nueva entidad; al Dr. Isidoro Sánchez Vega (CPUN) por el préstamo de material adicional; al Dr. Jim Solomon (MO) por confirmar la presencia del tipo en el herbario MO; y a la Bióloga Roxana Aguirre por la preparación de la excelente ilustración que forma parte de esta investigación.

\section{Literatura citada}

Bonpland A. \& A. de Humboldt. 1817. Nova Genera et Especies Plantarum. Tomo II. Lutetiae Parisiorum, Sumtibus Librariae Greco-Latino-Germanice, via dicta FossesMontmartre, No 14.

Epling C. \& C. Jativa. 1963. Supplementary notes on American Labiatae. VIII. Brittonia 15(4): 366-376.

Epling C. \& C. Jativa. 1966. Supplementary notes on American Labiatae. IX. Brittonia 18(3): 255-265.

Epling C. \& C. Jativa. 1968. Supplementary notes on American Labiatae. X. Brittonia 20(4): 295-313.

Fernández-Alonso J.L. 2008a. Estudios en Labiatae de Colombia VII. Salvia yukoyukparum, nueva especie y primer representante de la Sección Tomentellae en Colombia. Novon 18: $38-42$.

Fernández-Alonso J.L. 2008b. Estudios en Labiatae - VI. Hibridación en el género Salvia en Colombia y su interés horticultural. Caldasia.30 (1):.21-48.

Granda A. 2010. Salvia hunzikeri (Lamiaceae), una nueva especie de los Andes del Perú. Rev. peru. biol. 17(2): 151-154.

Holmgren P. K., N. H. Holmgren \& L. C. Barnett. 1990. Index Herbariorum. Part. I: The Herbaria of the World. $8^{\text {th }}$. ed. The New York Botanical Garden, Bronx, New York. U.S.A.

Macbride F. 1960. Labiatae. En Flora of Peru. Field Museum of Natural History, Botany 13(5/2): 721-829.

Rodríguez E. \& R. Rojas. 2002. El Herbario: Administración y Manejo de Colecciones Botánicas. Edit. por R. Vásquez M., Missouri Botanical Garden, St. Louis, U.S.A.

Rodríguez M. 2006. Lamiaceae endémicas del Perú. En El libro rojo de las plantas endémicas del Perú. Ed.: B. León et al. Rev. peru. biol. Número especial 13(2): 371-379.

UICN. 2001. Categorías y Criterios de la Lista Roja de la UICN. Versión 3.1. Preparado por la Comisión de Supervivencia de Especies de la UICN. UICN, Gland, Suiza y Cambridge, Reino Unido.

Weigend M. 2002. Observations on the Biogeography of the Amotape-Huancabamba Zone in Northern Peru. In: K. Young et al., Plant Evolution and Endemism in Andean South America. Bot. Review 68(1): 38-54.

Weigend M. 2004. Additional observations on the biogeography of the Amotape-Huancabamba zone in Northern Peru: Defining the South-Eastern limits. Rev. peru. biol. 11(2): 127-134.

Zarucchi J. L. 1993. Lamiaceae. In: Brako, L. \& Zarucchi, J.L. (eds.). Catálogo de las Angiospermas y Gimnospermas del Perú. Monographs in Systematic Botany from the Missouri Botanical Garden 45: 579-590. 